\title{
Transplantation of ovarian granulosa-like cells derived from human induced pluripotent stem cells for the treatment of murine premature ovarian failure
}

\author{
TE LIU ${ }^{1,2}$, QIONG LI ${ }^{1}$, SUWEI WANG ${ }^{1}$, CHUAN CHEN $^{1}$ and JIN ZHENG ${ }^{2}$ \\ ${ }^{1}$ Central Laboratory, Shanghai Geriatric Institute of Chinese Medicine; \\ ${ }^{2}$ Department of Traditional Chinese Medical Gynecology, Longhua Hospital, \\ Shanghai University of Traditional Chinese Medicine, Shanghai 200031, P.R. China
}

Received May 22, 2015; Accepted April 4, 2016

DOI: $10.3892 / \mathrm{mmr} .2016 .5191$

\begin{abstract}
Premature ovarian failure (POF) is a common cause of female infertility, for which there are currently no ideal treatments or medications. Furthermore, apoptosis of ovarian granulosa cells (OGCs) is an important mechanism underlying the decline in ovarian reserve and function. In the present study, several cellular growth factors and hormones were used to induce the differentiation of human induced pluripotent stem cells (iPSCs) into ovarian granulosa-like cells (OGLCs) in vitro. Immunohistochemical staining demonstrated that OGLCs derived from iPSCs strongly expressed granulosa cell markers, including anti-Müllerian hormone, inhibin $\alpha$, inhibin $\beta$ and follicle-stimulating hormone receptor, but did not express stem cell markers, including octamer-binding transcription factor 4, SRY (sex determining region Y)-box 2, Nanog and stage-specific embryonic antigen-4 12 days post-induction. In addition, a mouse model of POF was generated by cyclophosphamide treatment. Subsequently, iPSC-derived OGLCs were transplanted into the POF mice (OGLCs-iPSCs-POF group) in vivo. Results indicated that, compared with the control group (POF mice treated with phosphate-buffered saline), the growth state of OGLCs was markedly improved, and mature follicles could be detected in the ovarian tissue of the OGLCs-iPSCs-POF group. Immunohistochemical staining demonstrated that iPSC-derived
\end{abstract}

Correspondence to: Professor Jin Zheng, Department of Traditional Chinese Medical Gynecology, Longhua Hospital, Shanghai University of Traditional Chinese Medicine, 365 Xiangyang Road, Shanghai 200031, P.R. China

E-mail: jinzheng2013@126.com

Professor Chuan Chen, Central Laboratory, Shanghai Geriatric Institute of Chinese Medicine, Longhua Hospital, Shanghai University of Traditional Chinese Medicine, 365 Xiangyang Road, Shanghai 200031, P.R. China

E-mail: chuanchen9453@126.com

Key words: premature ovarian failure, induced pluripotent stem cells, ovarian granulosa-like cells, transplantation
OGLCs transplanted into POF mice not only exhibited substantial growth in murine ovarian tissues, but also strongly expressed OGC markers. Furthermore, enzyme-linked immunosorbent assays indicated that the levels of the hormone estradiol in peripheral blood samples were significantly enhanced following transplantation of iPSC-derived OGLCs into POF mice. Furthermore, ovarian tissue weight was significantly higher in the OGLCs-iPSCs-POF group compared with in the control group, and the number of atretic follicles in OGLCs-iPSCs-POF mice was significantly reduced, as compared with in the control mice. These results suggest that OGLCs derived from human iPSCs may not only effectively enhance OGC growth and repair damaged ovarian tissue, but may also maintain the ovarian tissue niche, promoting follicular development and maturation in a mouse model of POF.

\section{Introduction}

Premature ovarian failure (POF) occurs in women before the age of 40, and is characterized by amenorrhea, infertility, low estrogen levels, high gonadotropin levels and a lack of mature follicles (1-4). POF is a common cause of female infertility, the pathogenesis of which is complex. Various defects, including genetic mutations, autoimmune disorders, infections and endocrine dysfunction may result in POF (1-4). Furthermore, patients with POF often exhibit abnormal karyotypes, follicle-stimulating hormone (FSH) abnormalities, autoimmune ovarian failure and ovarian reserve depletion (1-4). The mechanisms underlying POF are largely unknown; however, previous studies have demonstrated that a decline in ovarian reserve is closely associated with the occurrence and development of POF (1-4). Apoptosis of ovarian granulosa cells (OGCs) is an important mechanism underlying the decline of ovarian reserve and function (DORF). OGCs have a supporting and estrogen-mediated regulatory role in the maturation and development of oocytes, and maintain hormonal balance in the ovarian niche, in order to promote oocyte maturation via autocrine and paracrine mechanisms (1-4). Previous studies have indicated that when OGCs undergo a certain degree of apoptosis, ovarian atresia is stimulated, which leads to DORF and POF (2-4). Therefore, in order to maintain ovarian function, oocyte viability and 
normal ovulation, high quality OGCs are required. In 2006, Takahashi and Yamanaka reported that induced pluripotent stem cells (iPSCs) could be induced to differentiate from mouse fibroblasts via the addition of four transcription factors: SRY (sex determining region Y)-box 2 (Sox2), octamer-binding transcription factor 4 (Oct4), Kruppel-like factor 4 and c-Myc (5). iPSCs share salient characteristics with embryonic stem cells; however, they are generated by reprogramming somatic cells through the forced expression of key transcription factors (2,3,5-9). iPSCs are capable of self-renewal and pluripotent differentiation, and express specific biomarkers, including stage-specific embryonic antigen-4 (SSEA), Oct4, Sox 2 and Nanog. In addition, iPSCs express alkaline phosphatase (AKP) and telomerase at high levels $(5,9)$. iPSCs not only provide a valuable model for the in vitro study of the regulation and underlying mechanisms of cell development, but also provide abundant material for cellular genetic research $(2,3,5-9)$. In our previous study, microRNA-17-3p was used to guide the differentiation of human iPSCs into hormone-sensitive ovarian surface epithelium (OSE)-like cells in vitro. Ovarian weight and plasma estradiol (E2) levels were increased over time following transplantation of these OSE-like cells into a mouse model of POF. Furthermore, these OSE-like cells survived within the POF mouse ovaries for $\geq 14$ days, and expressed cytokeratin 7 and estrogen receptor $\beta$ proteins (3). These findings suggested that iPSCs may provide a significant investigational resource for emerging concepts in cell therapy and regenerative medicine by exploitation of their pluripotent characteristics. In the present study, human iPSCs were induced to differentiate into ovarian granulosa-like cells (OGLCs) in vitro. Subsequently, these cells were transplanted into POF mice. The present study aimed to evaluate the therapeutic potential of such cells for the treatment of POF at the pathological and molecular level.

\section{Materials and methods}

Human iPSCs culture and differentiation. DYR0100 human iPSCs (SCSP-1301) were obtained from Professor Xiaoyan Ding (Stem Cell Bank, Chinese Academy of Sciences, Shanghai, China). All experiments using DYR0100 iPSCs were approved by the Shanghai University of Traditional Chinese Medicine Research Ethics Board (Shanghai, China). The DYR0100 iPSCs were cultured in Dulbecco's modified Eagle's medium:F12 (1:1) supplemented with $15 \%$ KnockOut $^{\mathrm{TM}}$ Serum Replacement, $1 \mathrm{mM}$ sodium pyruvate, $2 \mathrm{mM}$ L-glutamine, $0.1 \mathrm{mM}$ nonessential amino acids, $0.1 \mathrm{mM}$ beta-mercaptoethanol, penicillin (25 U/ml)-streptomycin (925 mg/ml), Reconstituting Human Basic Fibroblast Growth Factor (15 ng/ml), Reconstituting Human Epidermal Growth Factor $(15 \mathrm{ng} / \mathrm{ml})$. The aforementioned cell culture reagents were purchased from Gibco (Thermo Fisher Scientific, Inc., Waltham, MA, USA). Cells were incubated in a humidified incubator containing $5 \% \mathrm{CO}_{2}$ at $37^{\circ} \mathrm{C}$. DYR0100 iPSCs were induced to differentiate into OGLCs for 12 days, according to a multistage protocol using appropriate cell growth factors and hormones (Table I). Briefly, the iPS cells were stimulated with all-trans-retinoic acid (atRA) for $48 \mathrm{~h}$, then atRA was removed. The iPS cells were cultured in fresh medium supplemented with E2, FSH and human growth hormone $(\mathrm{hGH})$ for $48 \mathrm{~h}$. The iPS cells were then cultured in fresh medium supplemented with anti-Müllerian hormone
(AMH), estradiol, FSH and hGH for 48 h. Finally, the iPS cells were cultured in fresh medium supplemented with AMH, estradiol, inhibin $\alpha$, inhibin $\beta$ and transforming growth factor- $\beta$ for $96 \mathrm{~h}$.

Murine model of $P O F$ and in vivo xenograft experiments. As described in our previous studies (2-4), hebetic female C57BL/6 mice ( $n=30$; age, 5 weeks) were obtained from the Shanghai University of Traditional Chinese Medicine with approval from the Institutional Animal Care and Use Committee. To generate a murine model of POF, the mice were administered a single intraperitoneal injection of $70 \mathrm{mg} / \mathrm{kg}$ cyclophosphamide (Sigma-Aldrich, St. Louis, MO, USA). The mice were divided into two groups: A negative control group $(n=15)$ treated with phosphate-buffered saline (PBS), and an experimental group $(n=15)$ transplanted with iPSC-derived OGLCs. A total of 1 week after the POF models were established, each mouse received an injection of $50 \mu$ l cells $\left(\sim 1 \times 10^{7}\right.$ cells $\left./ \mu 1\right)$, which were stained with DiI red fluorescent dye (Beyotime Institute of Biotechnology, Hangzhou, China), or $50 \mu \mathrm{l}$ PBS. The final experiments in both groups were conducted 21 days post-transplantation. All mice were sacrificed by cervical dislocation following this treatment and additional experiments were conducted.

AKP assay. An AKP assay kit (Beyotime Institute of Biotechnology) was conducted, according to the manufacturer's protocol. Briefly, the cells were washed twice with PBS and fixed in 4\% paraformaldehyde (Sigma-Aldrich) for $20 \mathrm{~min}$. Dye solution was added to the cells, which were incubated at $37^{\circ} \mathrm{C}$ for 60 min until the AKP-positive cells were stained deep blue. The staining reaction was terminated following the addition of PBS. Digital images were captured using a fluorescence microscope (DMI3000; Leica Microsystems, Inc., Buffalo Grove, IL, USA).

Immunofluorescent staining. As previously described $(3,4)$, the cultured cells or ovarian tissue sections were washed three times with PBS and were fixed with $4 \%$ paraformaldehyde for $30 \mathrm{~min}$. After blocking with blocking solution (Beyotime Institute of Biotechnology), the cells were incubated with primary antibodies (Table II) overnight at $4^{\circ} \mathrm{C}$, followed by a 30 min incubation with cyanine 3- or fluorescein isothiocyanate-conjugated goat anti-rabbit immunoglobulin G antibodies (1:200; cat. no. C0992; Sigma-Aldrich) and $5 \mu \mathrm{g} / \mathrm{ml} \mathrm{4',6-diamidino-2-phenylindole}$ (Sigma-Aldrich) at room temperature. Subsequently, the cells and tissues were thoroughly washed with Tris-buffered saline containing 0.5\% Tween-20 (Sigma-Aldrich) and were visualized by fluorescence microscopy (DMI3000; Leica Microsystems, Inc.).

Hematoxylin-eosin (H\&E) staining. As previously described $(3,4)$, fresh ovarian tissues were washed three times with PBS and were fixed with $4 \%$ paraformaldehyde for $30 \mathrm{~min}$. The tissue specimens were then dehydrated in a graded ethanol series, vitrified in xylene, and paraffin embedded. Serial $6-\mu \mathrm{m}$ sections were prepared and stained with $H \& E$. Images were captured using a DMI3000 microscope (Leica Microsystems, Inc.).

Enzyme-linked immunosorbent assay (ELISA). Mouse E2 and FSH ELISAs (Shanghai XiTang Biotechnology Co., Ltd., 
Table I. Human growth factors and hormones used to induce differentiation.

Factor

All-trans-retinoic acid

Estradiol

Anti-Müllerian hormone

Follicle stimulating hormone

Inhibin $\alpha$

Inhibin $\beta$

Transforming growth factor- $\beta$

Human growth hormone
Company (location)

Concentration $(\mathrm{ng} / \mathrm{ml})$

Table II. Primary antibodies, their source and dilutions.

Antibody (cat. no.)

Rabbit anti-human/mouse Oct 4 (sc-9081)

Rabbit anti-human/mouse Sox2 (sc-20088)

Rabbit anti-human/mouse Nanog (sc-33759)

Rabbit anti-human/mouse SSEA-4 (sc-21704)

Rabbit anti-human/mouse inhibin $\alpha$ (sc-30146)

Rabbit anti-human/mouse inhibin $\beta$ (sc-50288)

Rabbit anti-human/mouse AMH (sc-67287)

Rabbit anti-human/mouse FSHR (sc-13935)
Company (location)

Dilution

Santa Cruz Biotechnology, Inc. (Dallas, TX, USA)

$1: 100$

Santa Cruz Biotechnology, Inc.

$1: 100$

Santa Cruz Biotechnology, Inc.

$1: 100$

Santa Cruz Biotechnology, Inc.

$1: 100$

Santa Cruz Biotechnology, Inc.

$1: 100$

Santa Cruz Biotechnology, Inc.

$1: 100$

Santa Cruz Biotechnology, Inc.

$1: 100$

Santa Cruz Biotechnology, Inc.

$1: 100$

Oct 4, octamer-binding transcription factor 4; Sox2, SRY (sex determining region Y)-box 2; SSEA-4, stage-specific embryonic antigen-4; $\mathrm{AMH}$, anti-Müllerian hormone; FSHR, follicle-stimulating hormone receptor.

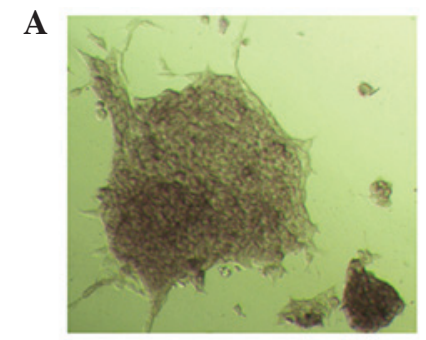

D

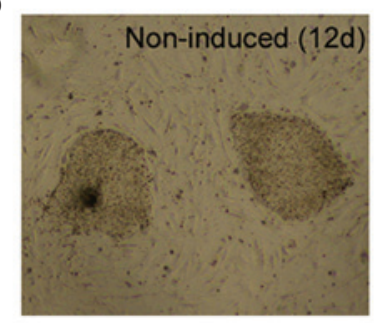

B
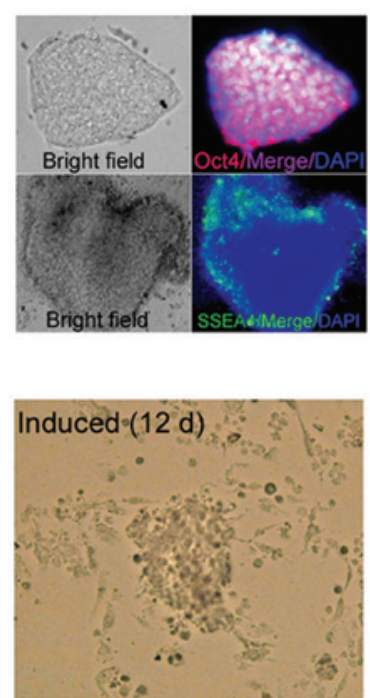

C Time:

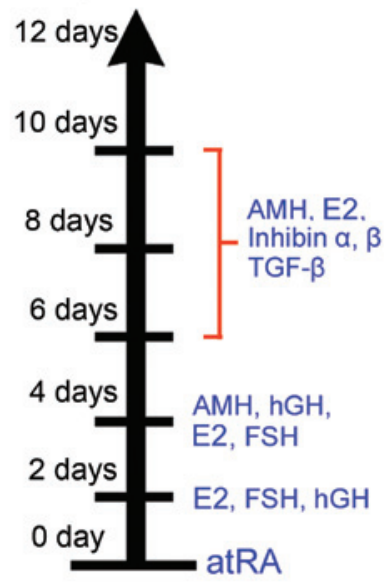

Figure 1. 'Stemness' of induced pluripotent stem cells (iPSCs) following induction. (A) Alkaline phosphatase (AKP) staining assay indicated that iPSCs exhibited deep blue staining. Original magnification, x200. (B) Immunofluorescence staining indicated that iPSCs highly expressed octamer-binding transcription factor 4 (Oct4) (red fluorescence) and stage-specific embryonic antigen-4 (SSEA4) (green fluorescence) proteins. Original magnification, x200. (C) Various cell growth factors and hormones were used at various time points to induce iPSCs to differentiate into ovarian granulosa-like cells. (D) Phenotype of iPSCs during induction. Original magnification, x200. AMH, anti-Müllerian hormone; E2, estradiol; TGF- $\beta$, transforming growth factor- $\beta$; hGH, human growth hormone; FSH, follicle-stimulating hormone; atRA, all-trans-retinoic acid.

Shanghai, China) were conducted according to the manufacturer's protocols and our previous studies $(3,4)$. Mouse blood plasma was obtained by retro-orbital blood collection (4). Briefly, a microhematocrit tube (BD Biosciences, Franklin Lakes, NJ, 


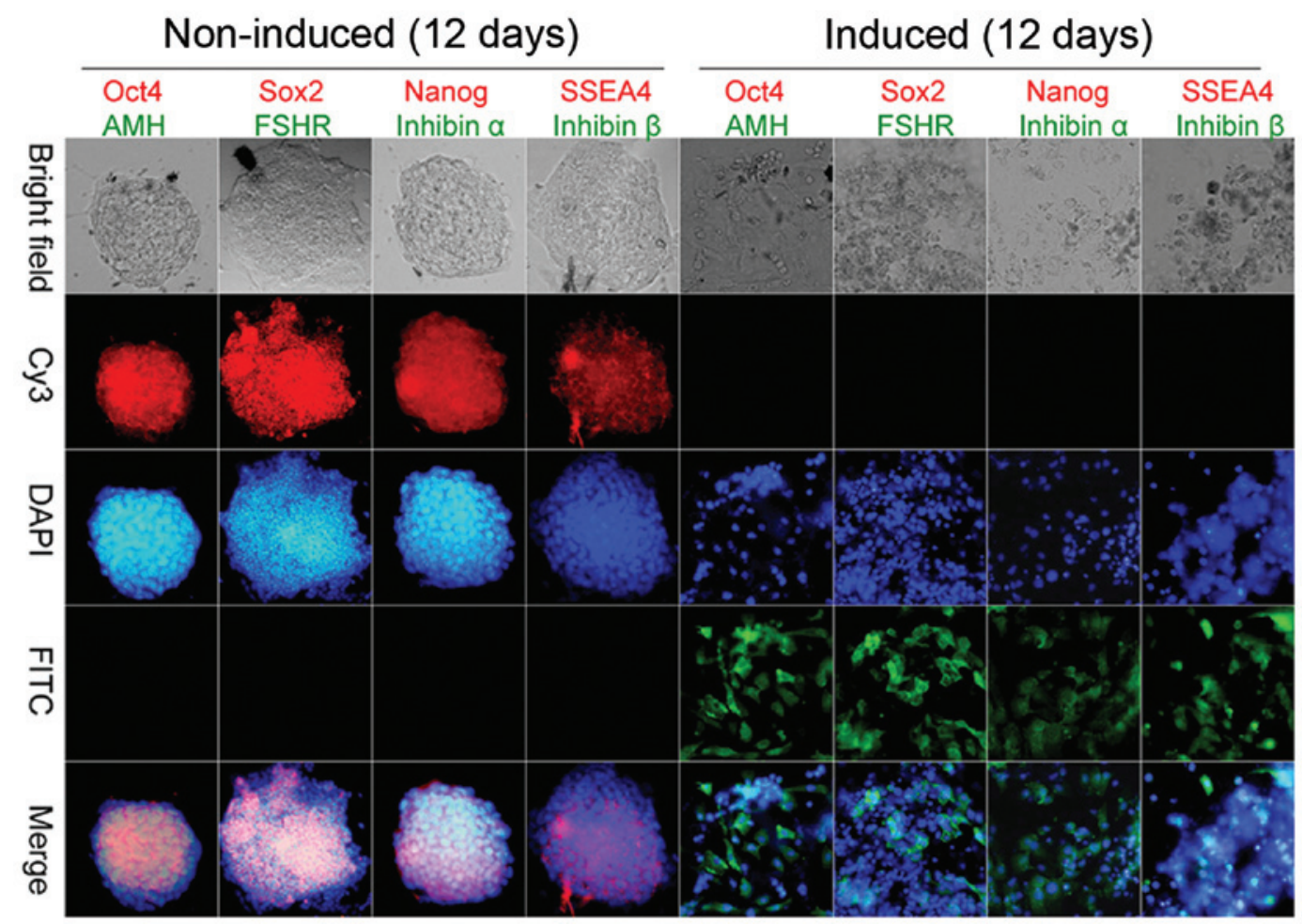

Figure 2. Immunofluorescence staining. Immunohistochemical staining indicated that ovarian granulosa-like cells derived from induced pluripotent stem cells strongly expressed ovarian granulosa cells markers [anti-Müllerian hormone (AMH), inhibin $\alpha$, inhibin $\beta$ and follicle-stimulating hormone receptor (FSHR)], but not stem cell markers [octamer-binding transcription factor 4 (Oct4), SRY (sex-determining region Y)-box 2 (Sox2), Nanog and stage-specific embryonic antigen-4 (SSEA4) 12 days post-induction. Each sample was stained for two markers with the color of the fluorescence indicated by the color of the word. Original magnification, x200. Cy3, cyanine 3; DAPI, 4',6-diamidino-2-phenylindole; FITC, fluorescein isothiocyanate.

USA) was inserted into the mouse lateral canthus at 30 degree angle to the side of the head. Approximately $100 \mu \mathrm{l}$ blood was collected from each mouse into heparin-coated blood collection tubes (BD Biosciences). Mouse blood was centrifuged at $453 \mathrm{x} \mathrm{g}$ at $4^{\circ} \mathrm{C}$ for $10 \mathrm{~min}$ and the supernatant was collected. Briefly, $100 \mu 1$ mouse E2 or FSH standards at the following concentrations: E2, 8,000, 4,000, 2,000, 1,000, 500, 250 and $125 \mathrm{pg} / \mathrm{ml}$; FSH, 10,000, 5,000, 2,500, 1,250, 625, 312 and $156 \mathrm{pg} / \mathrm{ml}$, or diluted mouse plasma were added to anti-E2 or anti-FSH pre-coated microtest wells and were incubated for $60 \mathrm{~min}$. The plates were then washed three times with PBS, and horseradish peroxidase-conjugated antibodies were added, followed by an enzymatic substrate. Optical absorbance was determined at a wavelength of $450 \mathrm{~nm}$ using a BioTek Synergy Mx plate reader (BioTek Instruments, Inc., Winooski, VT, USA).

Statistical analysis. Each experiment was performed as least three times, and results are presented as the mean \pm standard error of the mean. Data was analyzed using GraphPad Prism software (version 5.0; GraphPad Software, Inc., La Jolla, CA, USA). Where applicable, differences were evaluated by Student's t-test. $\mathrm{P}<0.05$ was considered to indicate a statistically significant difference.

\section{Results}

iPSCs can be induced to differentiate into OGLCs in vitro. Pluripotent stem cell biomarkers were detected prior to induction. Cellular immunostaining detected high expres-
Table III. Ovarian weight, hormone levels and the number of atretic follicles.

\begin{tabular}{lcc}
\hline Parameter & OGLCs-iPSCs-POF & PBS-POF \\
\hline E2 $(\mathrm{pg} / \mathrm{ml})$ & $158.6 \pm 42.2^{\mathrm{a}}$ & $87.45 \pm 19.0$ \\
FSH $(\mathrm{pg} / \mathrm{ml})$ & $435.7 \pm 184.1^{\mathrm{a}}$ & $971.9 \pm 218.5$ \\
Ovarian weight $(\mathrm{mg})$ & $3.7 \pm 1.1^{\mathrm{a}}$ & $2.3 \pm 1.0$ \\
Atretic follicles & $4 \pm 2^{\mathrm{a}}$ & $9 \pm 2$
\end{tabular}

${ }^{\mathrm{a}} \mathrm{P}<0.05$ vs. PBS-POF group. E2, estradiol; FSH, follicle-stimulating hormone; OGLCs, ovarian granulosa-like cells; iPSCs, induced pluripotent stem cells; POF, premature ovarian failure; PBS, phosphate-buffered saline.

sion levels of AKP, Oct4 and SSEA-4 (Fig. 1A and B). Subsequently, various cell growth factors (transforming growth factor- $\beta$ and human growth hormone) and hormones [E2, anti-Müllerian hormone (AMH), inhibin $\alpha$ and inhibin $\beta$ ] were used to induce iPSCs to differentiate into OGLCs at various time points (Fig. 1C). Following 12 days of induction, morphological observations indicated that the original clone-like iPSCs had successfully differentiated into fibroblast-like cells (Fig. 1D). Cell immunofluorescence indicated that prior to induction, iPSCs expressed high levels of pluripotent stem cell markers, including Oct4, Sox2, Nanog and SSEA-4, but did not express OGC markers, including $\mathrm{AMH}, \mathrm{FSH}$ receptor (FSHR), inhibin $\alpha$ and inhibin $\beta$. 
A

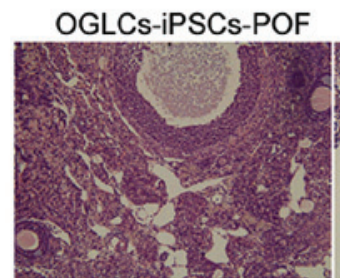

B

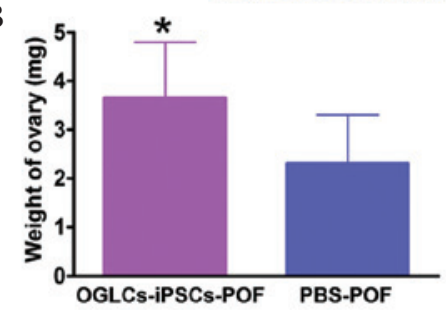

D

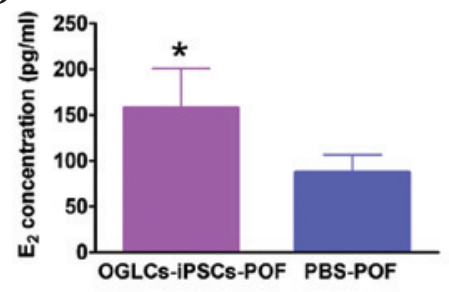

PBS-POF

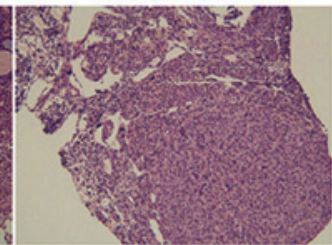

C

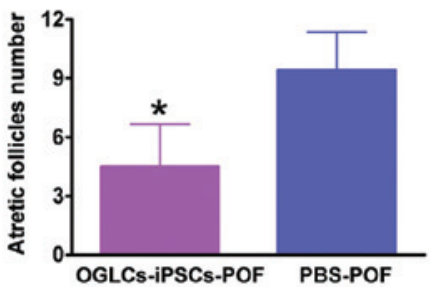

$\mathbf{E}$

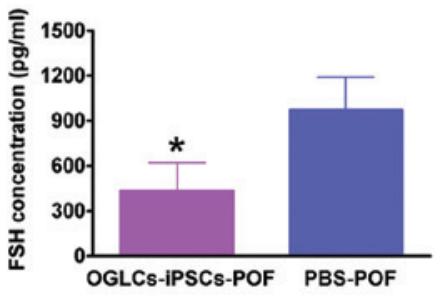

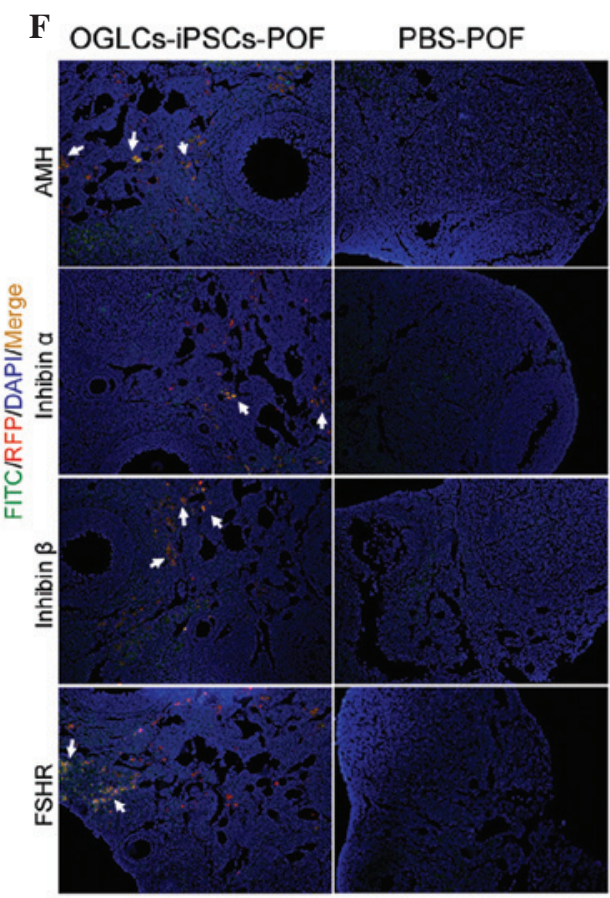

G

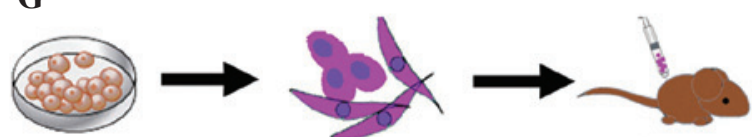

iPSCs

Oct4(+)

OGLCs-iPSCs

Sox2(+)

E2(+)

Transplant

$\mathrm{Nanog}(+)$

$\mathrm{AMH}(+)$

into POF mouse

Inhibin $\alpha, \beta(+)$

Figure 3. Effects of induced pluripotent stem cell (iPSC)-derived ovarian granulosa-like cells (OGLCs) transplanted into a mouse model of premature ovarian failure (POF). (A) Hematoxylin-eosin staining and pathological analysis revealed that iPSC-derived OGLCs improved the ovarian tissue niche, reduced the number of atretic follicles, and increased the number of normal follicles. OGLCs-iPSCs-POF, POF mice transplanted with iPSC-induced OGLCs (experimental group); PBS-POF, POF mice treated with PBS (control group). Original magnification, x200. (B) Ovarian tissue weight was elevated in the OGLCs-iPSCs-POF group compared with in the control group. (C) Number of atretic follicles in the OGLCs-iPSCs-POF group was significantly reduced. (D) Plasma estradiol (E2) levels were determined by enzyme-linked immunosrobent assay (ELISA); E2 levels were higher in the OGLCs-iPSCs-POF group compared with in the PBS-POF group. (E) Plasma follicle-stimulating hormone (FSH) levels were determined by ELISA; FSH levels were lower in the OGLCs-iPSCs-POF group compared with in the PBS-POF group. Data are presented as the mean \pm standard error of the mean. ${ }^{*} \mathrm{P}<0.05$ vs. the PBS-POF group; $n=15$. (F) Immunofluorescence assay indicated that iPSC-derived OGLCs transplanted into POF mice (red fluorescence) expressed OGC biomarkers (green fluorescence) in ovarian tissue. White arrows represent cells with red fluorescence merged with green fluorescence. Original magnification, x200. (G) iPSCs induction and transplantation process. AMH, anti-Müllerian hormone; Oct4, octamer-binding transcription factor 4; Sox 2, SRY (sex-determining region Y)-box 2; FSHR, FSH receptor; FITC, fluorescein isothiocyanate; RFP, red fluorescent protein; DAPI, 4'6-diamidino-2-phenylindole.

Conversely, following induction, the expression levels of OGC markers were elevated in granulosa-like cells derived from iPSCs (Fig. 2). These results suggest that iPSCs may be induced to differentiate into granulosa-like cells in vitro, following treatment with a combination of hormones and growth factors. 
OGLCs derived from iPSCs can reverse POF in mice. A total of 21 days post-transplantation the mice were sacrificed. In the control group (PBS-POF), ovarian pathological analysis indicated that normal follicles were almost absent. Ovarian stromal tissue and OGCs exhibited hallmarks of apoptosis and atrophy. Furthermore, ovarian tissue presented varying degrees of bleeding and serious follicular atresia (Fig. 3A). However, compared with the PBS-POF group, in the experimental group (OGLCs-iPSCs-POF), ovarian pathological analysis revealed that ovarian tissue contained some normal mature follicles, with a significant reduction in the numbers of atretic follicles. In addition, the growth state of the OGCs was markedly improved (Fig. 3A). Ovarian tissue weight was increased in the OGLCs-iPSCs-POF group compared with in the PBS-POF group $(\mathrm{P}<0.05$; Fig. 3B). The number of atretic follicles in the OGLCs-iPSCs-POF mice was reduced, as compared with in the PBS-POF mice ( $\mathrm{P}<0.05$; Fig. 3C). Furthermore, detection of hormone levels in murine peripheral blood samples indicated that the levels of E2 were elevated in the OGLCs-iPSCs-POF group, whereas the levels of FSH were decreased (Fig. 3D and E; Table III). Furthermore, in the OGLCs-iPSCs-POF group, immunofluorescence indicated that iPSC-derived OGLCs transplanted into POF mice (visualized by red fluorescence) expressed OGC biomarkers (AMH, inhibin $\alpha$, inhibin $\beta$, FSHR; green fluorescence) in ovarian tissue (Fig. 3F). These results suggest that iPSCs-derived OGLCs were capable of repairing ovarian injury and enhancing ovarian function.

\section{Discussion}

DORF is a typical characteristic and initial stage of POF (1). Ovarian reserve and function encapsulates the quantity and quality of follicles in the ovarian cortex, follicular growth capacity, ovarian development and oocyte formation (1-4). In addition, ovarian reserve and function is a measure of female reproductive endocrine functionality and fertility potential (1-4). The quality of OGCs directly influences ovarian reserve and function, and poor quality OGCs may eventually lead to the occurrence of POF (1-4). OGCs are the most immediate factor influencing ovarian function and follicular maturation (2-4). The present study aimed to determine whether ovarian tissue functionality can be enhanced or even restored following transplantation of OGCs (1-4). The present study evaluated techniques to induce differentiation of iPSCs into OGLCs in vitro. The results indicated that iPSCs could be induced to differentiate into OGLCs following treatment with numerous cell growth factors and hormones. Post-induction, iPSC-derived OGLCs exhibited the morphology of fibroblast cells, rather than that of embryonic stem cell clones. Furthermore, iPSC-derived OGLCs strongly expressed OGC biomarkers, but did not express markers characteristic of iPSCs. Subsequently, iPSC-derived OGLCs were transplanted into POF mice, and were revealed to express OGC biomarkers in ovarian tissue, improve ovarian tissue maturation status and further maintain follicular growth. These findings suggested that iPSC-derived OGLCs may be capable of re-establishing ovarian function. In addition, although the number of OGLCs observed in the ovarian tissues of POF mice were low, they served a role in ovarian repair. Growth hormones are crucial for the maintenance of ovarian follicular development, including $\mathrm{AMH}$, inhibin $\alpha$ and inhibin $\beta$. The prevalence and maturation of follicles is not only modulated by the pituitary gonadal axis, but is also influenced by specific cytokines in the ovarian niche. AMH, inhibin $\alpha$ and inhibin $\beta$ are members of the transforming growth factor $\beta$ superfamily, which are secreted by OGCs and exert an important role in ovarian maturation and recruitment. In addition, iPSCs-derived OGLCs did not induce teratoma formation and were relatively quiescent in vivo in the POF mice (Fig. 3G).

The results of the present study indicated that iPSC-derived OGLCs exhibited complete differentiation in vitro and were stable when transplanted into normal mice. In conclusion, the present study provides a novel insight regarding the treatment of POF. iPSC-derived OGLCs were able to enhance ovarian function in a murine model of POF, resulting in a reversal of ovarian dysfunction and thereby promoting follicular maturation.

\section{Acknowledgements}

The present study was supported by a grant from the National Natural Science Foundation of China (grant no. 81273794) to Jin Zheng. In addition, this work was supported by grants from the National Natural Science Foundation of China (grant no. 81202811), the Project funded by China Postdoctoral Science Foundation (grant no. 2014M550250), and the Shanghai Municipal Health Bureau Fund (grant no. 20124320) to Te Liu. The authors declare no potential conflicts of interest.

\section{References}

1. Vujović S, Ivović M, Tancić-Gajić M, Marina L, Barać M, Arizanović Z, Nenezić A, Ivanisević M, Micić J, Sajić S and Micić D: Premature ovarian failure. Srp Arh Celok Lek 140: 806-811, 2012.

2. Liu T, Huang Y, Guo L, Cheng W and Zou G: CD44+/CD105+ human amniotic fluid mesenchymal stem cells survive and proliferate in the ovary long-term in a mouse model of chemotherapy-induced premature ovarian failure. Int J Med Sci 9: 592-602, 2012.

3. Liu T, Qin W, Huang Y, Zhao Y and Wang J: Induction of estrogen-sensitive epithelial cells derived from human-induced pluripotent stem cells to repair ovarian function in a chemotherapy-induced mouse model of premature ovarian failure. DNA Cell Biol 32: 685-698, 2013.

4. Liu T, Huang Y, Zhang J, Qin W, Chi H, Chen J, Yu Z and Chen C: Transplantation of human menstrual blood stem cells to treat premature ovarian failure in mouse model. Stem Cells Dev 23: 1548-1557, 2014.

5. Takahashi K and Yamanaka S: Induction of pluripotent stem cells from mouse embryonic and adult fibroblast cultures by defined factors. Cell 126: 663-676, 2006.

6. Kiskinis E and Eggan K: Progress toward the clinical application of patient-specific pluripotent stem cells. J Clin Invest 120: 51-59, 2010.

7. Liu T, Chen Q, Huang Y, Huang Q, Jiang L and Guo L: Low microRNA-199a expression in human amniotic epithelial cell feeder layers maintains human-induced pluripotent stem cell pluripotency via increased leukemia inhibitory factor expression. Acta Biochim Biophys Sin (Shanghai) 44: 197-206, 2012.

8. Liu T, Cheng W, Huang Y, Huang Q, Jiang L and Guo L: Human amniotic epithelial cell feeder layers maintain human iPS cell pluripotency via inhibited endogenous microRNA-145 and increased Sox 2 expression. Exp Cell Res 318: 424-434, 2012.

9. Liu T, Zou G, Gao Y, Zhao X, Wang H, Huang Q, Jiang L, Guo L and Cheng W: High efficiency of reprogramming CD $34^{+}$cells derived from human amniotic fluid into induced pluripotent stem cells with Oct4. Stem Cells Dev 21: 2322-2332, 2012. 